\title{
Magnetopause Boundary Layers
}

\author{
Rickard LUNDIN \\ Swedish Institute of Space Physics, Box 812, S-98128 Kiruna, Sweden
}

(Received August 27, 1990; Revised December 4, 1990)

\begin{abstract}
The formation of boundary layers inside and outside of the magnetopause discontinuity of the Earth is a consequence of mass and momentum transfer processes through the magnetopause. The existence of these boundary layers is well recognized nowadays. However, the physics related to for instance the transfer of energy through the magnetopause into the inner magnetosphere is still a matter of controversy. In the reconnection scenario the magnetospheric boundary layer constitutes a "transport region" whilst the boundary layer model assumes that it constitutes a dynamo/generator region. This report will review recent developments in this field of research and address some of the main controversies related to the steady/nonsteady transfer of energy into the magnetosphere. Particular emphasis will be put on observations related to the coupling of the magnetospheric boundary layers to the low-altitude ionospheric dissipation region.
\end{abstract}

\section{Definitions and Overview}

The magnetopause boundary layers constitute layers of plasma lying immediately adjacent to the magnetopause-outside or inside of the magnetic surface discontinuity defined as the magnetopause. Despite more than 20 years of intense experimental and theoretical research, utilizing different in-situ spacecraft measurement techniques, the nature and consequences of these boundary regions in space is still debated.

The magnetopause boundary layer can be divided up into three parts, the magnetopause layer, the magnetosheath boundary layer, and the magnetospheric boundary layer. The magnetopause layer - the magnetopause proper - is a layer of plasma that contains the magnetic discontinuity separating the interplanetary magnetic field from the planetary (terrestrial) magnetic field. Notably, this magnetic discontinuity also marks a plasma discontinuity that separates plasmas of distinctly different characteristics.

The magnetopause layer has a width which is determined by the thickness of the current sheet required to maintain the jump in magnetic field orientation on both sides of the magnetopause. Discussions about the magnetopause current system were first made by CHAPMAN and FERRARO (1931) and they are often referred to as the Chapman-Ferraro currents.

The magnetopause layer has been simulated from single-particle theory by WILLIS (1975), the magnetopause thickness then given by the ion Larmor radius of the magnetosheath plasma (gyro-reflection of the magnetosheath flow). RUSSELL and ELPHIC (1979) determined on the basis of ISEE $1 / 2$ measurements an average magnetopause layer thickness of $600-800 \mathrm{~km}$, i.e., a few times the proton gyroradius. 
A magnetosheath boundary layer was proposed on theoretical grounds by COWLEY (1980), the magnetosheath boundary layer being a direct consequence of the open model magnetosphere. The discovery of flux transfer events (FTEs) in the magnetosheath (RUSSELL and ELPHIC, 1978) and the associated outflow of magnetospheric plasma on magnetosheath FTEs (e.g. SCHOLER et al., 1982) marks in a way also the first identification of the magnetosheath boundary layer. However, it has long been known from particle orbit theory of drifting particles that there must be a continuous loss of particles at the dayside magnetopause, also in a closed magnetic field model. These particles constitute a layer of magnetospheric particles in the magnetosheath. It has been argued that many of the FTE observations may simply be due to wave motions in the magnetopause, encountering the natural escape layer of magnetospheric particles (SIBECK et al., 1988). Thus there are two distinguishably different ways of interpreting the formation of a magnetosheath boundary layer; one based on magnetospheric field lines interconnected to interplanetary field lines (direct access), the other based on particle drifts through the dayside magnetopause layer (diffusion).

The magnetospheric boundary layer, frequently referred to as simply the boundary layer, was first identified by HONES et al. (1972) from IMP 6 data. Succeeding this discovery was a number of studies from IMP 6/7 (e.g. EASTMAN et al., 1976), HEOS (e.g. ROSENBAUER et al., 1975), ISEE 1/2 (e.g. PASCHMANN et al., 1979) and Prognoz-7 (e.g. LundiN et al., 1981), classifying the boundary layer from a morphological and topological point of view.

The magnetospheric boundary layer has been given various names such as: The lowlatitude boundary layer (LLBL), the entry layer (EL), the exterior cusp (EC), and the plasma mantle (PM) (see Fig. 1). The LLBL is often, if not predominantly, located on closed field lines while PM is located on open field lines.

The magnetic connection of the magnetospheric boundary layer was debated immediately after the boundary layer discovery. VASYLIUNAS (1975) described the boundary layer as the region of magnetic field reversal in an open magnetic field topology. On the other hand, EASTMAN et al. (1976) considered the LLBL to be the site of energy and momentum transfer predominantly on closed magnetic field lines. These two different viewpoint reflect an ongoing cause-effect controversy in what controls the solar wind mass and energy transfer into the magnetosphere. If we for a start leave the magnetic connection aside and look for a working definition of the magnetospheric boundary layer based on the plasma properties we may use the following definition:

The magnetospheric boundary layer is a region of plasma immediately inside the magnetopause which has predominantly magnetosheath characteristics and tailward/antisunward flow.

Figure 1 is a schematic picture summarizing the discussion about the magnetopause boundary layers, the top figure describing the boundary layers from a physics point of view and the bottom figure mapping out the various magnetopause boundary layers discussed in the literature. The top figure illustrates how plasma diffuses through/penetrates the magnetopause from the solar wind and the magnetosphere into the magnetopause boundary layers.

This report will primarily focus on processes taking place in the magnetospheric boundary layer. The context of the discussions will be in the frame of the intrinsic properties and processes within the magnetopause boundary layers, specifically the local transfer of energy, mass and momentum. It will be assumed in this report that detailed discussions on reconnection and flux transfer events (FTEs) are made by others in this volume (e.g. 

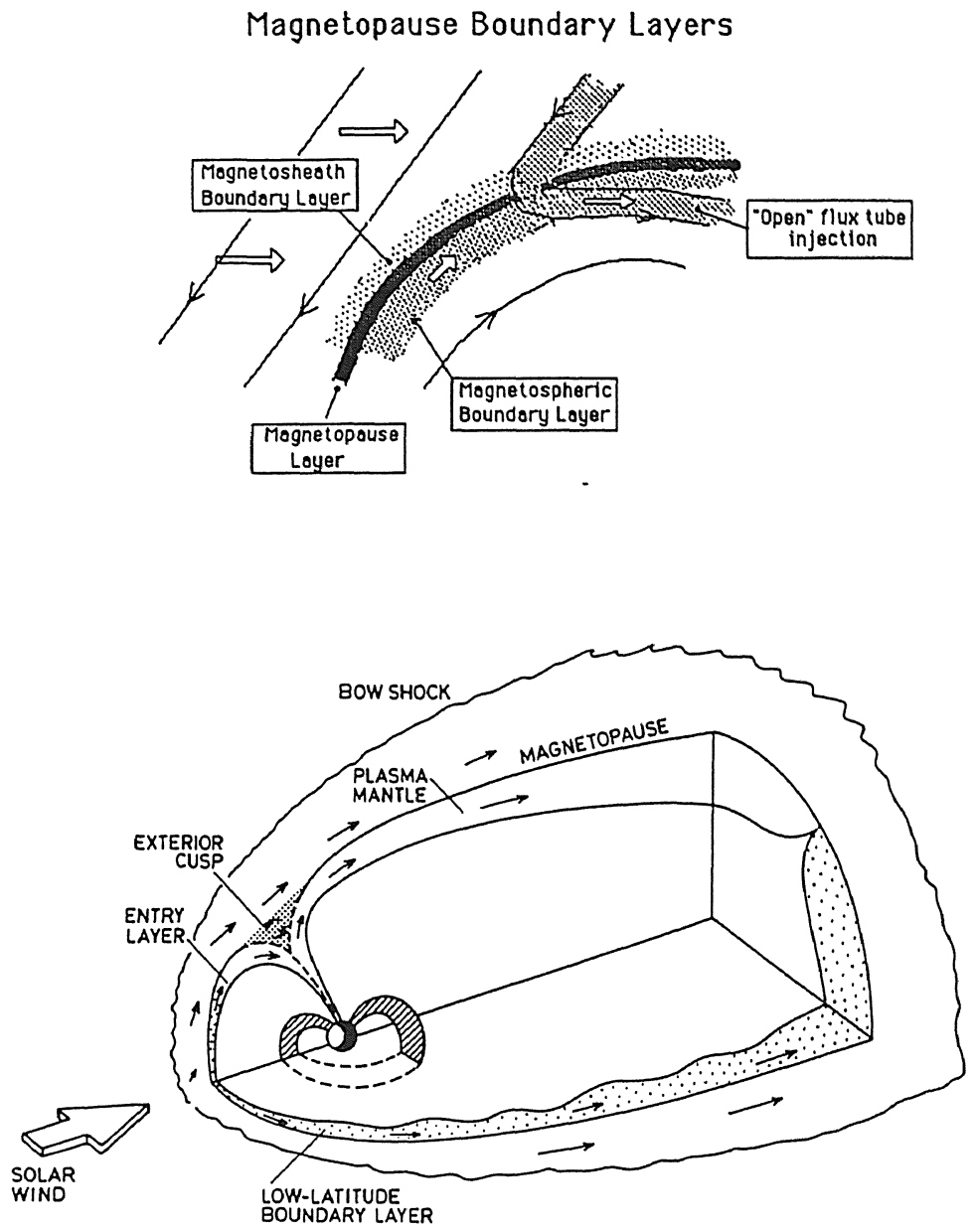

Fig. 1. The magnetopause boundary layers from a physical point of view (top). The Earths magnetosphere with its magnetospheric boundary layers (bottom).

SAUNDERS, 1991, this volume). The discussions on FTEs will be focussed on the expected plasma signature, the injection of magnetosheath plasma, rather than the magnetic signature of FTEs.

\section{Access of Solar Wind Plasma into the Magnetospheric Boundary Layer}

The magnetopause, the contact surface between the solar wind and the magnetospheric plasma, is often discussed from a magnetic field topology point of view rather than from a plasma or electric current point of view. Of particular interest is the connection of the interplanetary magnetic field (IMF) with the terrestrial magnetic field, denoted by the term merging/reconnection. Merging is a rather neutral concept, implying an opening of the magnetopause with associated access of solar wind plasma to magnetospheric field lines. On 
the other hand, reconnection can be considered as a current sheet disruption and is thus a process not necessarily associated with the dayside magnetopause only. Thus, the use of merging may be more appropriate when discussing plasma entry through the dayside magnetopause.

The topology of the magnetopause was recently described by STASIEWICZ (1990) using the magnetospheric model of TSYGANENKO (1987). From a magnetic topology point of yiew, assuming that polar cap field lines map to the solar wind only in the distant magnetotail, the northern and southern polar cusps are singularities mapping to the entire surface of the dayside magnetopause. However, a finite width of the magnetopause also means a finite width of the low-altitude cusp. Thus, the polar cusps should reflect the processes taking place within the dayside magnetopause. For instance, plasma crossing the magnetopause must traverse cusp field lines.

An example of a field line mapping of the magnetospheric boundary layer to the lowaltitude dayside auroral oval is depicted in Fig. 2 (STASIEWICZ, 1990), again based on the TSYGANENKO (1987) model. Although these kinds of mappings are done on basis of an

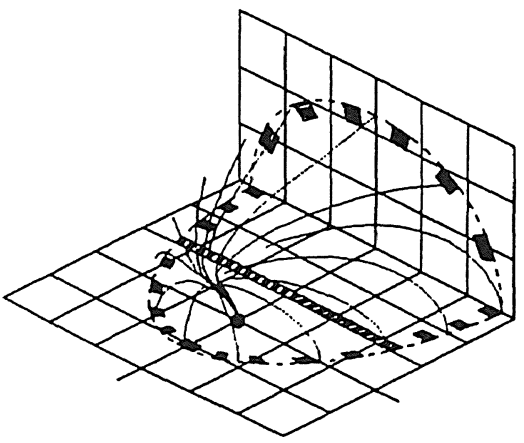

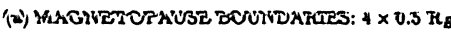

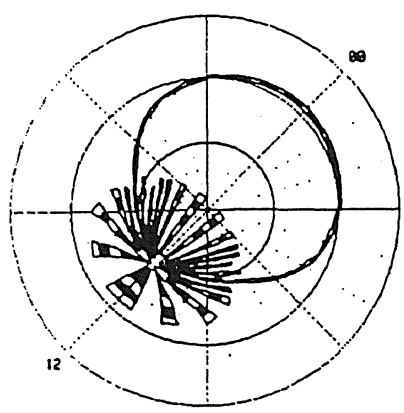

(b) IONOSPHERUC FOOTPRNTS

Fig. 2. (a) The magnetopause boundary layer in GSM coordinates and (b) its projection onto the ionosphere in polar coordinates (magnetic local time-magnetic latitude). Four layers with thickness $0.5 \mathrm{RE}$ each are painted with alternatively black and white. A cross-tail band maps to an ionospheric structure that simulated an auroral oval (after STASIEWICZ, 1990). 
averaged magnetic field model, unable to account for time dependent features in the magnetopause current sheet, they yet demonstrate that such projections may agree well with the dayside auroral morphology. Notice for instance that relatively simple gaps in the magnetopause boundary layer correspond astonishingly well with the "rayed" characteristics of auroral arcs observed near local noon (MENG and LUNDIN, 1986). Furthermore, the thin magnetopause layer expected to correspond to a narrow region near local noon - the cusp proper, expands when the magnetopause boundary layer widens.

The exterior cusp, from HEOS 2 measurements identified as a pocket of hot "stagnant" magnetosheath plasma in the distant part of the polar cusp (HAERENDEL et al., 1978, SCKOPKE et al., 1979), may possibly be considered part of the magnetopause layer. However, one may also argue that the exterior cusp is part of the magnetosheath boundary layer and is thus not necessarily on terrestrial field lines.

The cusp has long been known as a region of intense but relatively structureless precipitation of low-energy electrons of magnetosheath origin (e.g. BURCH, 1968, HEIKKILA and WinninghaM, 1971) near local noon. The cusp proper, defined as the region in continuous contact with the magnetosheath, constitutes a very narrow region at low altitudes (e.g. APARICIO et al., 1990). However, the region of enhanced/magnetosheath plasma is much broader. Figure 3 shows a schematic picture from a statistical study by KREMSER and LUNDIN (1990) that summarizes the findings within the dayside cusp/cleft region. Figure 3 also illustrates the three different ways in which solar wind plasma is believed to have access to the terrestrial magnetosphere: through continuous entry via the cusp proper, diffusive entry via the boundary cusp, and intermittent entry via the LLBL. The low-altitude counterpart of the LLBL-the cleft-represents the region where solar wind plasma is observed more intermittently from mechanisms providing impulsive entry through the dayside magnetopause. Such a plasma entry process, here termed plasma transfer events (PTEs), may be related with flux transfer events (FTEs) at the time of plasma injection.

A characteristic property of the cusp proper is the lack of plasma energization, while the boundary cusp/cleft display significant energization of both electrons and ions. This is an important and distinguishing difference that characterizes the different properties of the various magnetopause boundary layers to be discussed in the following sections.

Cusp /Cleft Structure

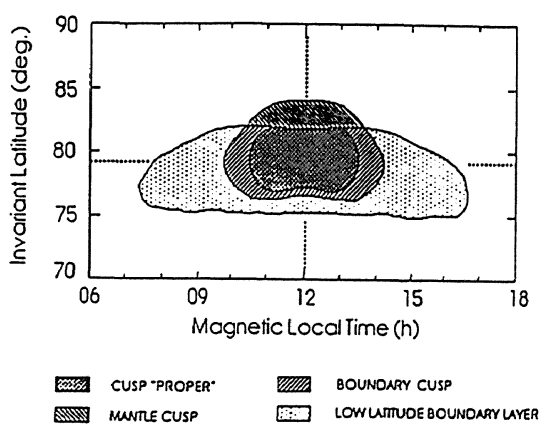

Fig. 3. Summary plot of the polar cusp and cleft from a statistical study by Viking (after KREMSER and LUNDIN, 1990). 
Intermittent injection of solar wind plasma through the magnetopause, PTEs, may occur as a result of an impulsive entry process (e.g. LEMAIRE, 1977) or through a spontaneous reconnection process denoted FTE (RUSSELL and ELPHIC, 1978). More recently NISHIDA (1989) explained spontaneous reconnection also for a northward IMF. Although impulsive entry and spontaneous reconnection may be considered different from a physical process point of view, they are yet in essence leading to the same result-PTEs.

Temporal injection of magnetosheath plasma in the dayside auroral oval, plasma transfer events (PTEs), have been reported from both sounding rockets and satellites. The first observations were presented by CARLSON and TORBERT (1980). On basis of the ion dispersion characteristics, frequently comprising several superimposed ion dispersion events, they were able to deduce the distance to the injection point to 7-19 $\mathrm{R}_{\mathrm{E}}$ away from the rocket altitude. This corresponds to somewhere between the entry layer (EL) and near equatorial region of the LLBL. Characteristics of plasma injection were also reported in situ from Prognoz-7 in the plasma mantle (PM) by LUNDIN and APARICIO (1982) and later also in the LLBL/EL by LUNDIN and DUBININ (1984).

Time dispersion features related with plasma injection are frequently found in data from Viking passes over the dayside auroral oval. An example of this is shown in Fig. 4. The characteristic time of the ion dispersion signature suggests here an injection of plasma some 9-11 $R_{E}$ upstream, i.e., into the high-latitude portion of the LLBL. An analysis of the energetic electron loss cones implied that at least the three equatormost injection structures occurred on closed magnetic field lines. The reader should note here the magnetic field signatures which are likely to be related to field aligned currents.

The magnetic field topology is important for distinguishing the various mechanisms proposed to be responsible for PTEs (impulsive entry, FTE, and solar wind pressure pulses). As indicated above, PTEs may occur on both open and closed field lines. However, according to a recent statistical study by WOCH and LUNDIN (1991a, b) they occur preferentially on closed field lines. This study also suggests that PTEs are a very common feature, with a frequency of occurrence along the dayside oval of $\approx 50 \%$. A substantial IMF- $B_{y}$ dependence

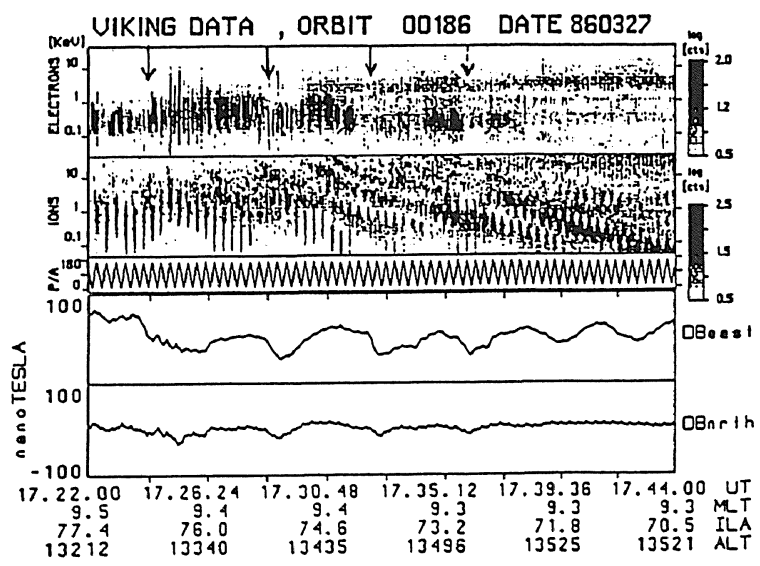

Fig. 4. Viking energy-time spectrogram illustrating several PTEs above the dayside oval, each arrow indicating the start of a plasma injection. 
but basically no IMF $B_{z}$ dependence was also found in this study.

Direct in-situ measurements of PTEs in the LLBL has been reported by LUNDIN and DUBININ (1984). Similar results by e.g. SCKOPKE et al. (1981) were, however, interpreted as a structure of the LLBL induced by Kelvin-Helmholtz instabilities. Since existing observations are based on single or at best two spacecraft, an unambiguous interpretation of PTEs as being isolated structures within the magnetosphere or attached to the magnetopause is virtually impossible. What can be concluded, though, is that according to the Viking study most PTEs are observed on closed field lines.

\section{General Plasma Properties within the Magnetospheric Boundary Layer}

The magnetospheric boundary layer represents in many aspects a transition between a high-density magnetosheath flow and a lower density/stagnant and hot magnetospheric plasma. The transition on a low-sampling rate or statistical basis marks a decreased flow and density region, frequently with a strong crossed field flow (EASTMAN et al., 1976). The flow has shear characteristics suggesting that a viscous interaction is present, possibly as a result of finite-Larmor radius effects (STASIEWICZ, 1989). A statistical analysis of the ionic composition also shows that the magnetospheric boundary layer may represent a relatively smooth transition between a magnetosphere plasma composition and a magnetosheath plasma composition. Figure 5 gives results from a statistical study using Prognoz-7 ion composition data taken from high-latitude passes near the GSM noon-midnight meridian $( \pm 3$
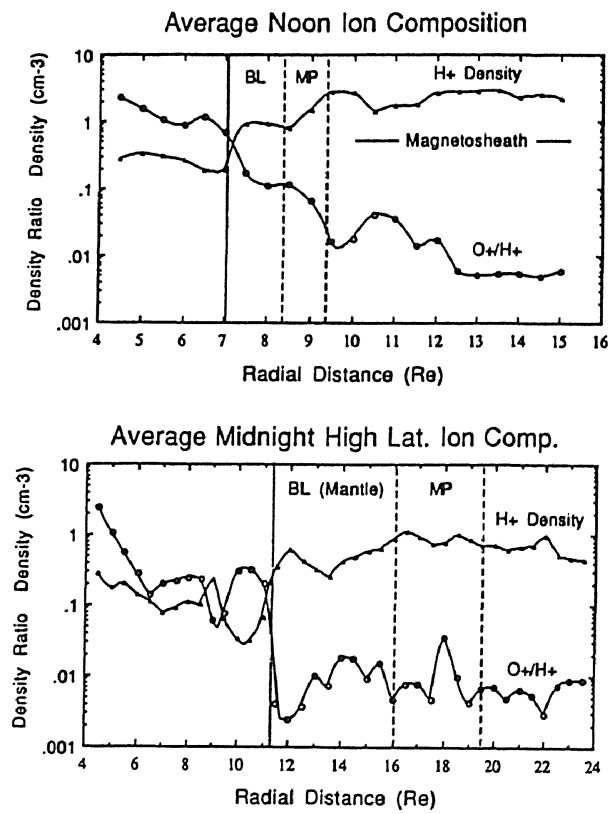

Fig. 5. Prognoz-7 observations of the average high latitude dayside and nightside $0.2-17 \mathrm{keV} / \mathrm{e}$ ion composition near the noon-midnight meridian ( \pm 3 hours), illustrating the transition from the magnetosphere to the magnetosheath of the heavy ion $\left(\mathrm{O}^{+}\right)$contribution. 
hours). Notice from this study that there is a difference between the dayside (LLBL) and nightside (PM) transition region in the heavy/ionospheric ion abundance. The dayside boundary layer is associated with a gradual transition between the magnetosphere and magnetosheath while on the nightside a more abrupt change between the inner magnetosphere and the boundary layer is present. Thus, although substantial heavy/ionospheric ion fluxes have been observed in the plasma mantle (e.g. LuNDIN et al., 1982), the average local midnight ( \pm 3 hours) mantle apparently contains on the average no more heavy ions than that further out in the magnetosheath. One implication of this is that the local midnight mantle is associated with only minor auroral energization processes, in agreement with its connection to the mantle cusp portion of the cusp proper. Another implication is that the mantle/lobe intersection represents the main composition boundary in the deep nightside, thus allowing little transport of ions across this boundary. The immediate consequence of the latter is that magnetosheath plasma does not convect across the tail lobe into the near Earth $\left(<20 R_{E}\right)$ nightside plasma sheet. This is in direct conflict with the hypothesis of the plasma mantle as the main contributor of plasma to the plasma sheet.

Another distinguishing difference between the plasma mantle and the LLBL is on the thickness of the boundary versus IMF $B_{z}$. From HEOS 2 SCKOPKE et al. (1976) showed that the mantle thickness is inversely proportional to the IMF $B_{z}$, a positive $B_{z}$ likely to be associated with no mantle at all. Conversely, the LLBL is shown to grow in width with increasing IMF $B_{z}$ (MITCHELL et al., 1987), the apparent average width being about 4 times
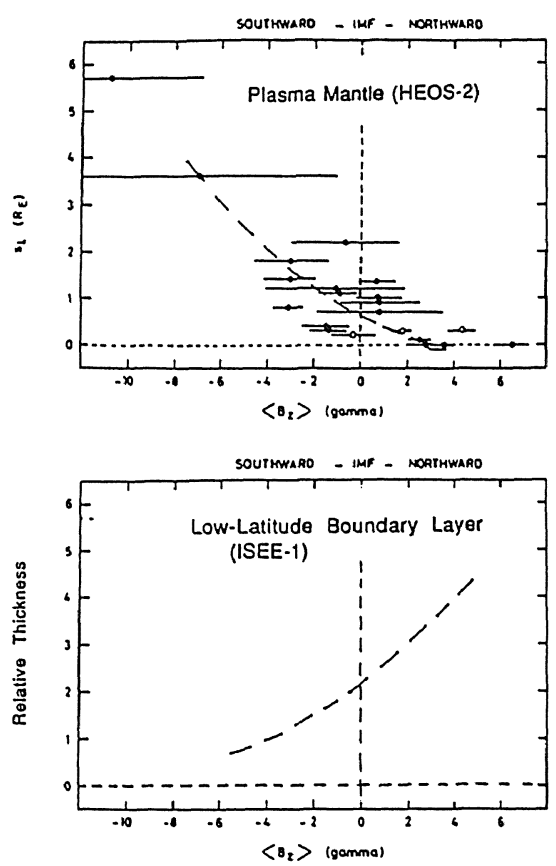

Fig. 6. Magnetospheric boundary layer thickness versus the IMF $B_{z}$, the top diagram illustrating the mantle thickness (after SCKOPKE et al., 1976), and the bottom diagram giving a schematic picture of the LLBL thickness (as interpreted from Mitchell et al., 1987). 
wider during a positive $B_{z}$ than during a negative $B_{z}$. Figure 6 display results from the statistical study by SCKOPKE et al. (1976) and a schematic picture illustrating the outcome of the study by MITCHELl et al. (1987). Notice that in the latter case no thicknesses were estimated but merely the duration time of the LLBL encounters.

While the average pass of the LLBL displays a smooth transition between the magnetosphere and the magnetosheath, data with a higher resolution shows that the LLBL is much more structured with "blobs" of magnetosheath plasma (SCKOPKE et al., 1981) and a poor mixing in terms of plasma composition (LUNDIN and DUBININ, 1984).

The radial direction of the $-(\boldsymbol{v} \times \boldsymbol{B})$ term in the dayside LLBL observation (e.g. LUNDIN and DUBININ, 1985), is consistent with a viscous-type of interaction in establishing the boundary layer. Thus, the boundary layer constitutes a flow essentially parallel with the external flow.

\section{The Boundary Layer as a Transport or Generator/Load Region}

The idea of a magnetospheric boundary layer as a driver for magnetospheric convection and currents stems back to AXFORD and HINES (1961) and COLE (1961). These rather simple conjectures of the boundary layer as a region of solar wind energy and momentum exchange were soon overpowered by the merging/reconnection hypothesis introduced by DUNGEY (1961).

In essence the boundary layer model that evolved from the primitive fluid picture by AXFORD and HINES (1961) was already emerging (COLE, 1961) as a dynamo/current circuit modelled propelled by the boundary layer plasma flow, a notion further developed by BOSTRÖM (1975) ROSTOKER and BOSTRÖM (1976), and SONNERUP (1980). A distinguishing and very important property of such a boundary layer model is that it puts the field aligned currents observed by low-altitude orbiting satellites (e.g. IJIMA and POTEMRA, 1978) into the right perspective. Conversely, the reconnection model by DUNGEY (1961) assumes a "simple" magnetic coupling of terrestrial magnetic field lines to the solar wind magnetic field, thus exposing the polar region ionosphere to solar wind electromagnetic forces. Circulation/convection within the magnetosphere is maintained by magnetic reconnection at the dayside magnetopause and in the magnetotail.

In the reconnection hypothesis the magnetospheric boundary layer is no more than a transport region for solar wind plasma on reconnected field lines. Conversely, in the boundary layer model this is the region where solar wind plasma is transferring energy and momentum into the topside ionosphere. Figure 7 gives a schematic summary view of the LLBL dynamo and the associated currents connected to the topside ionosphere as was originally proposed by COLE (1961) and later discussed by e.g. EASTMAN et al. (1976), HEIKKILA (1982), LUNDIN and EVANS (1985). If we compare this diagram with the model mapping of Fig. 2 we may note that the entire LLBL current system will close in the relatively narrow crescent shaped dayside cleft region. Notice that this model predicts an unbalanced internal/magnetospheric current system because the LLBL current system is assumed to be closed at the magnetopause on one side. This is in good agreement with observations of field aligned currents with lowaltitude orbiting satellites. IJMA and POTEMRA (1978) concluded for instance that the region 1 and region 2 currents are unbalanced near local noon, suggesting that the region 1 current is predominantly closed externally. They also noted that the region 1 currents are strongest in the dayside, independent of the disturbance level (AL index). This is thus an indication that the LLBL dynamo/generator on the average delivers the highest energy input into the 


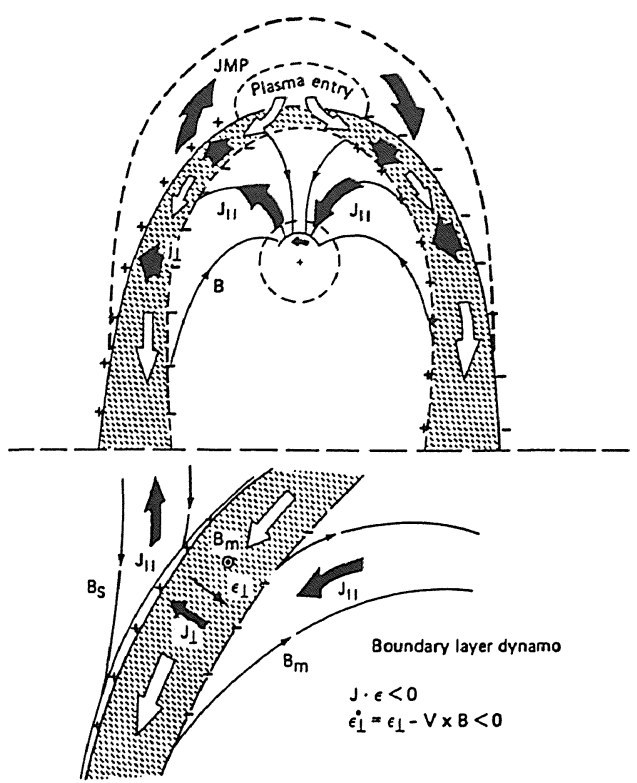

Fig. 7. A model of the magnetospheric boundary layer dynamo generated currents and fields (from LUNDIN and Evans, 1985). The lower panel gives a schematic model of the boundary layer polarization process. Notice that the (unbalanced) inner current circuit (region l) close in the cusp/cleft region (Figs. 2, 3).

magnetospheric current system. Notice, however, that a fraction of the dayside region 1 current must balance the observed dayside region 2 current (see e.g. ROSTOKER and MARESCHAL, 1982). The balanced system, closing via ionospheric Pedersen currents across the oval and possibly also via the polar cap, should play a larger (if not a dominant) role during substorm activity.

The polarization/dynamo voltage across the LLBL is as already mentioned (Fig. 7) driven by the plasma flow in the boundary layer. The lower part of Fig. 7 illustrates how the polarization current $J_{\perp}$ charges up the dynamo and provides an electric potential in the opposite direction of the current flow. Because the dynamo is magnetically connected to the resistive ionosphere, the dynamo becomes loaded. This causes a braking action of the dynamo plasma and a replenishment of charges in the polarization region. In effect this represents a conversion of boundary layer plasma kinetic energy into electromagnetic energy and heating/energization of ionospheric plasma. Notice also that a dynamo under load implies that there is a difference between the local electric field $(\varepsilon)$ and the $-(\boldsymbol{v} \times \boldsymbol{B})$ term. Thus, a heavily loaded dynamo is associated with a substantial difference between the motional EMF $\left(E=\int_{\boldsymbol{v}} \times \boldsymbol{B} \cdot \mathrm{d} s\right)$ and the induced potential $\left(U=\int \varepsilon \cdot \mathrm{d} s\right)$.

The polarization in the boundary layer is according to this model mainly radial in the dayside, inward at dusk and outward at dawn (Fig. 7). This orientation is also confirmed from measurements of the plasma $-(\boldsymbol{v} \times \boldsymbol{B})$ (e.g. EASTMAN et al., 1976, LUNDIN and DUBININ, 1984). Figure 8 gives two examples of Prognoz-7 passes in the dawn and dusk sector LLBL that illustrate this. Below each plot is a diagrammatic illustration of the orbit track with respect to the magnetopause pass. Note that the motional EMF of the dynamo plasma (solar 

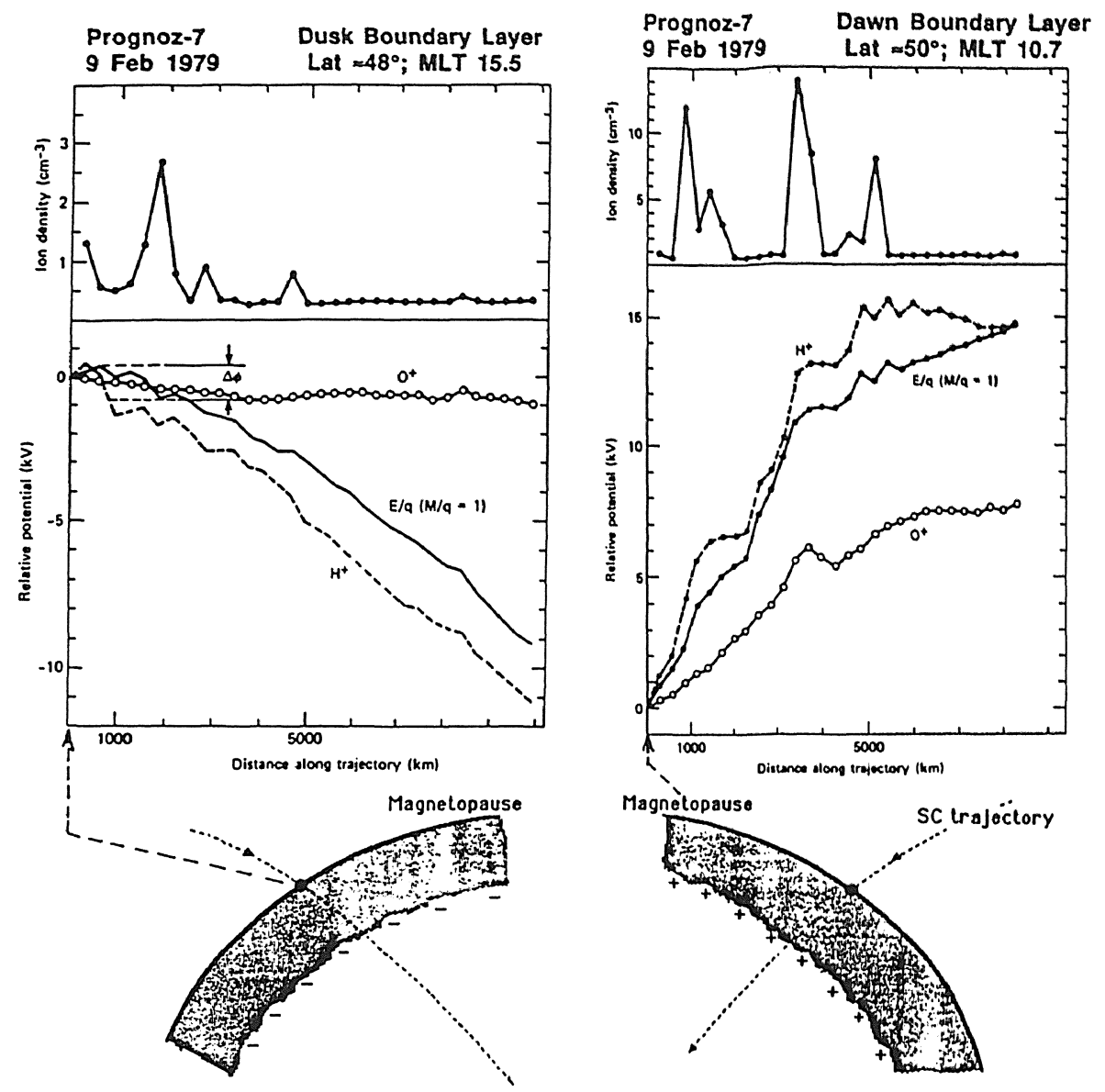

Fig. 8. Two Prognoz-7 crossings of the dawn and dusk boundary layer that give the integrated plasma $\boldsymbol{y} x$ $B$ along the spacecraft trajectory. The lower part illustrating the orbit track and the associated polarization/motional EMF deduced within the boundary layer.

wind protons) accumulated along the spacecraft track is in the same direction as predicted by the model in Fig. 8. The total EMF, adding dawn and dusk contributions together, is in the range of a few tens of $\mathrm{kV}$, i.e., a substantial fraction of the anticipated cross-tail potential. The fact that the EMF deduced from the $\mathrm{O}^{+}-(\boldsymbol{v} \times \boldsymbol{B})$ is substantially smaller than the $\mathrm{H}^{+}-(\boldsymbol{v}$ $\times B$ ) can be understood as two different plasma populations subjected to differences in e.g. partial pressure and inertia. However, one simple interpretation is to consider $\mathrm{O}^{+}$ions as "test particles" simply responding to the LLBL induced electric field while the $\mathrm{H}^{+}-(\boldsymbol{v} \times \boldsymbol{B})$ is due to a (longitudinal) pressure gradient within the MHD dynamo. The justification for such an interpretation is that $\mathrm{O}^{+}$ions constitutes a pre-existing low-density magnetospheric population, while $\mathrm{H}^{+}$ions predominantly belongs to a newly injected magnetosheath population. Thus, the integrated $\mathrm{O}^{+}-(\boldsymbol{v} \times \boldsymbol{B})$ would correspond to the electric potential $(U)$ over the boundary layer while the integrated $\mathrm{H}^{+}-(\boldsymbol{v} \times \boldsymbol{B})$ is equivalent with the motional 
EMF $(E)$, the difference $(E-U)$ corresponding to the ionospheric loading of the boundary layer dynamo.

The polarization of the boundary layer is also confirmed from electric field data (MOZER, 1984). However, instead of the tens of $\mathrm{kV}$ motional EMF estimated from the crossed-field flow, MOZER (1984) estimated the electric potential to be of the order a few $\mathrm{kV}$ only. HEIKKILA (1986) disputed this very low value by Mozer on basis of the one figure of the LLBL crossing published by MOZER (1984): Heikkila estimated a "radial" LLBL potential of $13 \mathrm{kV}$. However, even if this would represent an extreme case of boundary layer polarization there are yet three important conclusions that can be drawn here: First, observations indicates that the direction of the boundary layer electric field is radial, in good agreement with the boundary layer dynamo model. Secondly, a substantial deviation between the polarization and motional EMF voltage is expected when strong field aligned currents connected to the dynamo are driven through the conducting ionosphere (loaded dynamo). Thus, the electric field does not describe the total EMF within a dynamo under load. Yet a third conclusion is that the cases presented so far imply total (dawn-dusk) boundary layer EMFs in the 20-30 kV range, i.e., an order of magnitude higher than the few $\mathrm{kV}$ proposed by SONNERUP (1980) and inferred by MOZER (1984). Such high potentials are not much different from the dawn-dusk potentials measured over the polar cap. Thus, one may argue that the LLBL-potential indeed constitutes a substantial part of the dawn-dusk cross polar cap potential.

\section{Intrinsic Properties of the Boundary Layer Dynamo}

So far the boundary layer dynamo has been considered mainly from an external/loading point of view of the current circuitry, i.e., the dynamo represents a source of free energy provided to the upper atmosphere/ionosphere of the Earth. However, the intrinsic properties of the magnetospheric boundary layer are important for both understanding the physical mechanisms responsible for the conversion of free energy within the dynamo and the intrinsic parameters affecting such an energy conversion. Locally, the polarization is driven by a current as shown in Fig. 8, the external loading causing a difference in the electric field and the local $-(\boldsymbol{v} \times \boldsymbol{B})$. Provided a local pre-existing plasma is present in the boundary layer, momentum will also be transferred to the pre-existing plasma in the course of plasma injection. Thus, a pre-existing plasma will take up momentum from the injected/dynamo plasma in a collision-free process simply as a result of the electric field generated by the dynamo plasma. Such an "internal loading" of the dynamo can be described by a depolarization current as has been discussed by LUNDIN and DUBININ (1985). They showed that, using Ohm's law for determining the momentum transfer, one may obtain a velocity solution for a decelerating (dynamo) ion species and an accelerating (preexisting) ion species. Combining these velocities will lead to the following relation between the momentum absorbing ions (suffix 2) and the dynamo ions (suffix 1):

$$
v_{x 2}=v_{x 1}\left(1-\exp \left(-\frac{n_{1} m_{1}}{n_{2} m_{2}}\right)\right) \text {. }
$$

This relationship demonstrates the importance of internal loading, i.e., the momentum exchange between one plasma population flowing into another plasma population. Notice for instance from this relation that when the mass density of the injected plasma is much higher 
than the loading plasma $\left(n_{1} m_{1} \gg n_{2} m_{2}\right)$ the loading plasma will act as "test particles" moving with the convection speed $\left(v_{x 2} \approx v_{x 1}=\varepsilon_{y} / B_{z}\right)$. Conversely, a high internal loading would lead to a large difference in speed between the injected and loading plasma, eventually stopping the injected plasma.

A test of the momentum exchange in the boundary layer dynamo, incorporating both internal and external loading, was made by LUNDIN and DUBININ (1985), the result shown in Fig. 9. The parameters $R_{n}$ and $R_{\varepsilon}$ represent dimensionless parameters of densities and drift velocities $\left(R_{n}=\left(1+n_{1} / n_{2}\right) ; R_{\varepsilon}=\left(1+v_{x 2} / v_{x 1}\right)\right)$, where in this case the loading ions were assumed to be a mixture of cold and hot $\mathrm{O}^{+}$and $\mathrm{He}^{+}$, and the loading plasma drift was determined from $\mathrm{O}^{+}$. All protons were taken as injected plasma.

Under the assumption that the dynamo works in a steady state with the local plasma (e.g. $\mathrm{O}^{+}$and $\mathrm{He}^{+}$) moving with the convection speed (the heavy ions acting as test particles i.e. $\left.v_{x 2} \approx \varepsilon_{y} / B_{z}\right)$ we can define a loading parameter $K\left(K=v_{x 2} / v_{x 1} \approx \varepsilon_{y} / v_{x 1}\right)$ which then describes the external (ionospheric) load of the dynamo (right vertical axis). The diagram of Fig. 9 thus describes the internal load (horizontal axis) versus the external load (vertical axls).

The diagram of Fig. 9 demonstrates for instance that a high internal loading makes it difficult to distinguish between internal and external loading. Electric field measurements are then required. Conversely, a low internal loading would make it possible to determine the external loading from the difference in plasma drift between the injected and the local plasma However, the ion drift is not a reliable means of determining the convection speed - even for a low density population. This becomes clear from the general expression of the first order plasma drift:

$$
\underline{\nu}_{\perp}=\frac{\underline{B}}{B^{2}} \times\left[\underline{\varepsilon}+\frac{m d \underline{v}_{o}}{q d t}+\frac{\nabla P_{\perp}}{q n}\right]+\frac{\left(P_{I I}-P_{\perp}\right)}{q n}\left(\frac{\underline{B} \times \nabla B}{B^{3}}+\frac{\mu_{0}}{B^{2}} j_{\perp}\right)
$$

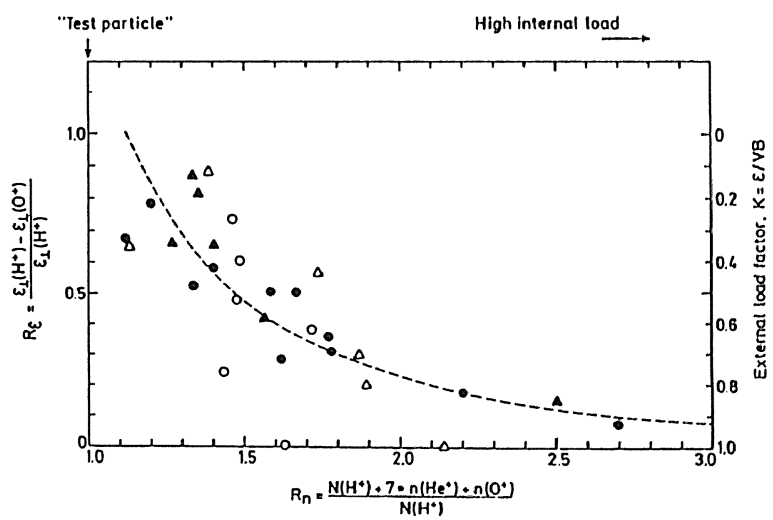

Fig. 9. A test of the momentum transfer in the boundary layer dynamo from normalized measured differences in plasma drift versus normalized plasma density differences. The horizontal axis illustrates the "internal loading" (by local plasma) and the vertical axis the "external loading" (by the ionospheric load) in the boundary layer dynamo. 
where the second term in the first bracket is the inertia drift, the third term is the pressure gradient drift, and the fourth term (not in bracket) is the anisotropy drift. Thus, besides convection there are four other terms that may be equally responsible for plasma drift. These terms become particularly important for ions. Notice that because the electric drift affects all species in the same way, the ion differential drift $\Delta v_{\perp}=v_{\perp 1}-v_{\perp 2}$ :

$$
\Delta v_{\perp}=(\Delta v)_{\nabla_{p}}+(\Delta v)_{a}+(\Delta v)_{i}
$$

only contains the differences in pressure drift, anisotropy drift, and inertia drift respectively. LUNDIN et al. (1987) also demonstrated, on basis of single point measurements, that partial pressure gradients could very well be responsible for the difference in ion drift observed along the spacecraft orbit.

So far, we have considered the magnetopause boundary layer mainly as a dynamo/decelerator for solar wind plasma. This is, as we already concluded, in contradiction with the presumption in the reconnection/merging hypothesis where e.g. the magnetospheric boundary layer is merely considered a transport region. There is, however, also evidence for that solar wind plasma may become accelerated close to the magnetopause (e.g. PASCHMANN et al., 1979, SONNERUP et al., 1981). We propose here that such an acceleration may take place in the thin magnetopause layer when the magnetopause current is parallel with the external electric field $(j \cdot \varepsilon>0)$, the magnetopause layer thus acting as a load for an externally applied electric field.

How frequent magnetopause plasma acceleration occurs is at present not very clear from the in-situ observations at the magnetopause. A few tens of cases have been reported from ISSE-1 and AMPTE. If the acceleration occurs at the magnetopause layer, it should also, for reasons discussed above, be observed in the cusp proper. Indeed, such cases also been reported from mid-altitude measurements (e.g. LYONS et al., 1987; LUNDIN, 1988). So far some 70 examples have been found in the Viking data, thus forming a good basis for statistical studies. In Fig. 10 we present a preliminary result from this study which shows that the

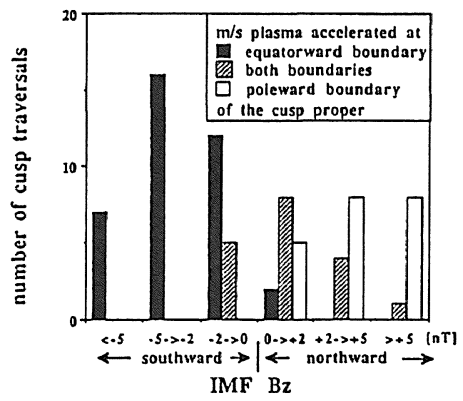

Fig. 10. Results from a statistical study of the current sheet acceleration near the cusp illustrating a good agreement with antiparallel merging, i.e., the acceleration mainly occurs for an antiparallel direction between the extemal (magnetosheath) and intemal magnetic field (after WOCH and LUNDIN, 1989). 
acceleration takes place in the equatorward boundary during southward $B_{z}$ and in the poleward boundary during northward $B_{z}$. This is thus in complete agreement with the antiparallel merging site being the location for current sheet acceleration. However, it is important to note that the current sheet acceleration takes place within a very limited region of the cusp/cleft. The dominating region of e.g. the boundary cusp and cleft (Figs. 2 and 3), maps to the LLBL that is associated with a massive transfer of solar wind/magnetosheath plasma energy into the ionosphere and the mid-altitude energization region.

\section{Conclusions}

The magnetopause boundary layers represent the transition between a high beta, flowing solar wind/magnetosheath plasma on one side and a hot, "stagnant", low beta magnetospheric plasma on the other, i.e., they separate plasmas of widely different characteristics. The transition can be divided into three types of magnetopause boundary layers:

- The magnetosheath boundary layer, lying outside the magnetopause and characterized by escaping magnetospheric plasma in the magnetosheath.

- The magnetopause layer, the magnetopause proper, defined by the thickness of the magnetopause current sheet.

- The magnetospheric boundary layer, lying inside the magnetopause and characterized by a plasma having predominantly magnetosheath characteristics and tailward/antisunward flow.

The magnetosheath boundary layer may be connected to sheath field lines or to newly opened terrestrial field lines, while the magnetopause layer is topologically connected to the terrestrial magnetic field via the polar cusp(s)/cusp proper. The cusp proper thus constitutes a region of the terrestrial magnetic environment which is in continuous contact with the solar wind/magnetosheath.

The magnetospheric boundary layers (EL, PM, LLBL) are topologically connected to a relatively narrow, crescent shaped, region of the ionosphere near local noon. Simultaneous with a widening of the magnetospheric boundary layers (MITCHELL et al., 1987), this region expands considerably during periods of strong northward IMF. According to the TSYGANENKO (1987) model the quiet time magnetic footprint of the magnetospheric boundary layers may even expand well into the nightside.

Solar wind plasma is believed to have access to the boundary layer via two mechanisms, diffusion through wave particle interactions (e.g. EVIATER and WOLF, 1968) or gyroviscosity (STASIEWICZ, 1989), or direct plasma entry (PTEs) through impulsive entry (LEMAIRE, 1977, HEIKKILA, 1982) or via FTEs (RUSSELl and ELPHIC, 1979). PTEs, observed in situ in the magnetospheric boundary layer as "blobs" of magnetosheath-like plasma, are also observed more deeply in the dayside magnetosphere from e.g. Viking. The majority of the Viking PTEs were observed on closed field lines (WOCH and LUNDIN, 1991b).

The magnetospheric boundary layer is characterized by a strong transverse plasma flow corresponding to $\approx 3-10 \mathrm{mV} / \mathrm{m}$. The perpendicular plasma flow along satellite traversals of the boundary layer corresponds to total motional EMFs up to several tens of $\mathrm{kV}$. The measured EMFs are thus in the range of the polar cap potentials measured from low-altitude satellites. The question of what magnetospheric boundary layer contributes most to the polar cap potential is a complex one that cannot be handled by simply eliminating one or the other. Only a few attempts have been made to evaluate the individual contribution from the two proposed processes for solar wind energy and momentum transfer (e.g. REIFF et al., 
1977, and COWLEY, 1982). One outcome of these investigations is that magnetically quiet periods appears to be dominated by viscous-like processes while active periods appears to be dominated by the reconnection process. However, it is probably more constructive to consider the specific energy transfer regions and their relation to ionospheric disturbances. For instance, the LLBL polarization is consistent with the overall current circuitry near local noon (e.g. the unbalanced region 1 current) and is thus predominantly related with dayside auroral activity. Contemporary magnetic field mapping models (e.g. TSYGANENKO, 1987) show, however, that the LLBL couples to the tail plasma sheet and thus considerably influence also the nightside current system and cross-tail potential. This illustrates some of the difficulties in evaluating the contributions from different sources to the polar cap potential.

The intrinsic plasma properties within the magnetospheric boundary layer, e.g. ion differential drift, are consistent with a dynamo under internal (local plasma) and external (ionospheric coupling) load. The ion differential drift can be understood from first order plasma drift theory as e.g. inertia drift and partial plasma pressure gradients in a magnetospheric boundary layer characterized by strong spatial/temporal gradient of the plasma properties. The implications of the ion differential drift is of course that "ideal" MHD $(\varepsilon+v$ $\times \boldsymbol{B}=0$ ) does not hold for ions in the boundary layer. The only species that potentially obeys "ideal" MHD there is thus electrons.

The deviation from "ideal" MHD is likely to be one reason for potential mismatches between the electric field/boundary layer potential and the cross-field plasma flow (motional EMF).

Although the dominating action is deceleration of magnetosheath plasma in the dayside magnetospheric boundary layer, local acceleration of plasma may also occur. Such an acceleration may occur inside the magnetospheric boundary layer as a result of the momentum transfer between the injected (magnetosheath) and the local/stagnant magnetosphere plasma and, secondly, in the magnetopause layer resulting from a local current sheet disruption/reconnection process $\left(j_{M P} \cdot \varepsilon>0\right)$. Such an acceleration has been observed both locally (ISEE-1, AMPTE) and at mid-altitudes (Viking).

\section{REFERENCES}

AXFORD, W. I. and C. O. Hines, A unifying theory of high latitude geophysical phenomena and geomagnetic storms, Can. J. Phys., 38, 1433, 1961.

Boström, R., Mechanisms for driving Birkeland currents, Physics of Hot Plasma in the Magnetosphere, edited by B. Hultqvist and L. Stenflo, Plenum Press, New York, p. 341, 1975.

Burch, J. L., Low-energy electron fluxes at latitudes above the auroral zone, J. Geophys. Res., 73, 3585, 1968.

Carlson C. W. and R. B. TORbert, Solar wind ion injections in the morning auroral oval, J. Geophys. Res., 85, 2903, 1980.

Chapman, S. and V. C. A. Ferraro, A new theory of magnetic storms, Terr. Mag. Atmosph. Elec., 36, 77, 1931.

Cole, K. D., On solar wind generation of polar magnetic disturbances, J. Astron. Soc., 4, 103, 1961.

Cowley, S. W. H., Plasma populations in a simple open magnetic field model magnetosphere, Space Sci. Rev., 26, 217, 1980.

COWLEY, S. W. H., The causes of convection in the earth's magnetosphere - A review of developments during the IMS, Rev. Geophys. Space Phys., 20, 531, 1982.

Dungey, J. W., Interplanetary fields and the auroral zone, Phys. Rev. Lett., 6, 47, 1961. 
Eastman, T. E., E. W. Hones, Jr., S. J. BAmE, and J. R. Asbridge, The magnetospheric boundary layer: Site of plasma, momentum and energy transfer from the magnetosheath into the magnetosphere, Geophys. Res. Lett., 3, 685, 1976.

Eviater, A. and R. A. Wolf, Transfer processes in the magnetopause, J. Geophys. Res., 1968.

Haerendel, G., G. Paschmann, N. Sckopke, H. Rosenbauer, and P. C. Hedgecock, The frontside boundary layer of the magnetosphere and the problem of reconnection, J. Geophys. Res., 83, 3195, 1978.

Heikkila, W. J., Impulsive plasma transport through the magnetopause, Geophys. Res. Lett., 9, 877, 1982.

Heikkila, W. J., Comment on electric field evidence on the viscous interaction at the magnetopause, by $F$. S. Mozer, Geophys. Res. Lett., 13, 233, 1986.

Heikkila W. J. and J. D. Winningham, Penetration of magnetosheath plasma to low altitudes through the dayside magnetic cusps, J. Geophys. Res., 76, 883, 1971.

Heikkila, W. J., Magnetospheric topology of fields and currents, in Magnetospheric Currents, AGU Geophysical Monograph, 28, A208, 1984.

Hones, E. W. Jr., J. R. Asbridge, S. J. Bame, M. D. Montgomery, S. Singer, and S.-I. Akasofu, Measurements of Magnetotail plasma flow with Vela 4B, J. Geophys. Res., 77, 5503, 1972.

IJima, T. and T. A. PotemRA, Large-scale characteristics of field aligned currents associated with substorms, J. Geophys. Res., 83, 599, 1978.

KREMSER, G. and R. LUNDIN, Average spatial distribution of energetic particles in the mid-altitude cusp/cleft region observed by Viking, J. Geophys. Res., 95, 5753, 1990.

Lemaire, J., Impulsive penetration of filamentary plasma elements into the magnetospheres of the Earth and Jupiter, Planet Space Sci., 25, 887, 1977.

LundiN, R., On the magnetospheric boundary layer and solar wind energy transfer into the magnetosphere, Space Sci. Rev., 48, 263, 1988.

Lundin, R., B. Hultevist, N. Pissarenko, and A. Zakharov, The plasma mantle: composition and other characteristics observed by means of the Prognoz-7 satellite, Space Sci. Rev., 31, 247, 1981.

LUNDIN, R. and B. APARICIO, Observations of penetrated solar wind plasma elements in the plasma mantle, Planet. Space Sci., 30, 81, 1982.

Lundin, R. and E. M. Dubinin, Solar wind energy transfer regions inside the dayside magnetopause -..Evidence for magnetosheath plasma penetration, Planet. Space Sci., 32, 745, 1984.

LUNDIN, R. and E. Dubinin, Solar wind energy transfer regions inside the dayside magnetopausc-... Accelerated heavy ions as tracers for MHD-processes in the dayside boundary layer, Planet. Space Sci., 33, 891-907, 1985.

Lundin, R. and D. S. Evans, Boundary layer plasmas as a source for high-latitude, early aftemoon, auroral arcs, Planet. Space Sci., 33, 1389, 1985.

Lundin, R., K. Stasiewicz, and B. HultQvist, On the interpretation of different flow vectors of different ion species in the magnetospheric boundary layer, J. Geophys. Res., 92, 3214, 1987.

Lyons, L. R., A. L. Vampola, and T. W. Speiser, Ion precipitation from the magnetopause current sheet, J. Geophys. Res., 92, 6147, 1987.

MenG, C.-I. and R. Lundin, Auroral morphology of the midday oval, J. Geophys. Res., 91, 1572, 1986.

Mitchell, D. G., F. Kutchio, D. J. Williams, T. E. Eastman, L. A. Frank, and C. T. Russell, An extended study of the low-latitude boundary layer on the dawn and dusk flanks of the magnetosphere, $J$. Geophys. Res., 92, 7394, 1987.

Mozer, F. S., Electric field evidence on the viscous interaction at the magnetopause, Geophys. Res. Lett., 11, 135, 1984.

NishidA, A., Can random reconnection on the magnetopause produce the low latitude boundary layer?, Geophys. Res. Lett, 16, 227, 1989.

Paschmann, G., B. U. Ö. Sonnerup, I. Papamastorakis, N. Sckopke, G. Haerendel, S. J. Bame, J. R. Asbridge, J. T. Gosling, C. T. Russell, and R. C. Elphic, Plasma acceleration at the earth's magnetopause: Evidence for reconnection, Nature Lond., 282, 243, 1979.

Reiff, P. H., T. W. Hill, and J. L. BurCH, Solar wind plasma injection at the dayside magnetospheric cusp, J. Geophys. Res., 82, 479, 1977.

Rosenbauer H., H. Grünwaldt, M. D. Montgomery, G. Paschmann, and N. Sckopke, HeOS 2 plasma observations in the distant polar magnetosphere: The plasma mantle, J. Geophys. Res., 80, 2723, 1975. 
ROSTOKER, G. and R. BOSTRÖM, A mechanism for driving the gross Birkeland current configuration in the auroral oval, J. Geophys. Res., 81, 235, 1976.

ROSTOKER, G. and M. MARESCHAL, Field aligned current and the auroral electrojets in the post-noon quadrant, J. Geophys. Res., 87, 9071, 1982.

RusSELL, C. T. and R. C. ELPHIC, ISEE observations of flux transfer events at the dayside magnetopause, Geophys. Res. Lett., 6, 33, 1979.

Sckopke, N., G. Paschmann, G. Haerendel, G. Papamastorakis, B. U. Ö. Sonnerup, S. J. Bame, T. G. FoRBES, E. W. HoNes Jr., and C. T. RusSELL, Structure of the low-latitude boundary layer, J. Geophys. Res., 86, 2099, 1981.

SCholer, M., D. Hovestadt, F. M. Ipavich, and G. Gloeckler, Energetic protons, alpha particles, and electrons in magnetic flux transfer events, J. Geophys. Res., 87, 2169, 1982.

SibECK, D. G., R. W. MCENTIRE, S. KRIMIGIS, and D. N. BAKER, The magnetosphere as a sufficient source for upstream ions on November 1, 1984, J. Geophys. Res., 93, 14328, 1988.

SONnerup, B. U. Ö., Theory of the low-latitude boundary layer, J. Geophys. Res., 85, 2017, 1980.

Sonnerup, B. U. Ö., G. Paschmann, I. Papamastorakis, N. Sckopke, G. Haerendel, S. J. Bame, J. R. AsBRidge, J. T. GosLiNG, and C. T. Russell, Evidence for magnetic field reconnection at the earth's magnetopause, J. Geophys. Res., 86, 10049, 1981.

Stasiewicz, K., A fluid Larmor radius model of the magnetopause layer, J. Geophys. Res., 94, 8827, 1989.

STASIEwiCZ, K., A gyroviscosity model of magnetopause merging, J. Geophys. Res., 1990 (in print).

TSYGANENKO N. A, Global quantitative models of the geomagnetic field in the cislunar magnetosphere for different disturbance levels, Planet Space Sci., 35, 1347, 1987.

Vasyliunas, V. M., Magnetic field line merging, Rev. Gephys. Space Phys., 13, 303, 1975.

WILLIS, D. M., The microstructure of the magnetopause, J. R. Astr. Soc., 41, 355, 1975.

WocH, J. and R. Lundin, Temporal magnetosheath plasma injection observed with Viking: A case study, to appear in Annales Geophysicae, 1991a.

WoCH, J. and R. Lundin, Signatures of transient boundary layer processes observed with Viking, submitted to $J$. Geophys. Res., $1991 \mathrm{~b}$.

WoCH., J. and R. Lundin, Magnetosheath plasma energization features observed in the mid-altitude cusp region, EOS, fall AGU, 1989. 